\title{
Consecuencias económicas en el Producto Interno Bruto del decrecimiento poblacional en los países que atraviesan la fase final de su transición demográfica
}

\author{
Mario Ernesto Donoso Correa ${ }^{1,2,3}$ (D), Paula Milena Cordero Cueva ${ }^{1}$, Nelson Federico Córdova \\ Gonzalez ${ }^{1}$ \\ ${ }^{1}$ Unidad de Ingeniería, Industria y Construcción, Universidad Católica de Cuenca, Cuenca, Ecuador. \\ 2 Programa VLIR-IUC en Migración y Desarrollo Local, Universidad de Cuenca-Universidades \\ Flamencas. \\ ${ }^{3}$ Facultad de Economía y Ciencias Administrativas, Universidad de Cuenca, Cuenca, Ecuador.
}

Autor para correspondencia: donoso.mario@gmail.com

Fecha de recepción: 25 de abril 2017 - Fecha de aceptación: 27 de noviembre 2017

\begin{abstract}
RESUMEN
Los países se han caracterizado por crecer demográficamente desde fines del siglo 18 hasta las últimas décadas del siglo 20, cuando algunas naciones más bien empezaron a estabilizarse e incluso a disminuir sus poblaciones, pasando por diferentes etapas del modelo de transición demográfica, debido a que las tasas de fertilidad son menores a los niveles de reemplazo de la población, ocasionando preocupación a los gobiernos que están conviviendo con este fenómeno, sobre todo en relación al posible declive de sus economías. Este estudio demuestra que si las economías logran mantenerse estables, entonces las consecuencias económicas del detrimento poblacional, pese a ser negativas desde el punto de vista macro, son positivas para los hogares, causando que el subempleo y desempleo disminuyan, generándose mejores ingresos y más consumo para las familias; y mientras más pronto los gobiernos se preparen y ajusten sus economías, las sociedades podrán adaptarse mejor a este cambio demográfico que inevitablemente ocurrirá en todas partes, a medida que avance el proceso de urbanización durante este siglo y el próximo.
\end{abstract}

Palabras clave: Modelo de transición demográfico, tasas de fertilidad, inmigración, despoblamiento, producto interno bruto.

\begin{abstract}
Countries have been characterized by demographic growth since the end of the 18th century until the last decades of the 20th century, when some nations began to stabilize and even decrease their populations. Those countries went through the different stages of the demographic transition model, because fertility rates decreased below the replacement levels of the population, causing concern to the governments of those countries, especially in relation to the possible decline of their economies. This study shows that rather the economic consequences of a decline in population, despite being negative from the macro point of view, are positive for households, if the economies manage to remain stable. The dwindling down of the population shall cause underemployment and unemployment to decrease, generating better income and more consumption for families. The sooner governments prepare and adjust their economies, societies will be better prepared to adapt to this demographic change that will inevitably occur everywhere, as the urbanization process advances during this century and the next one. Keywords: Demographic transition model, fertility rates, immigration, depopulation, gross domestic product.
\end{abstract}




\section{INTRODUCCIÓN}

La demografía es la ciencia que estudia las poblaciones (Poulalion, 1984) y por ende está relacionada con factores sociales, legales, políticos, culturales, psicológicos, ambientales y económicos que generan cambios en las variables demográficas: nacer, movilizarse y morir (Thomlinson, 1965). Durante mucho tiempo en los países menos desarrollados del planeta, ha sido la explosión demográfica un motivo de preocupación constante (Birdsall, Kelley \& Sinding, 2001). Sin embargo actualmente, en algunas naciones, la nueva preocupación es su despoblamiento (Morgan, 2003), por ende es importante entender la adaptación de estas sociedades a esta nueva realidad, así como describir las consecuencias de este declive demográfico, para proponer soluciones adecuadas que podrían implementarse.

La familia humana creció lentamente a una tasa constante; fue a partir de la Revolución Industrial (Ashton, 1997), durante los últimos 250 años que este incremento se suscitó de manera exponencial en todo el mundo, sobre todo desde mediados del siglo 20, cuando a partir del fin de la Segunda Guerra Mundial, los países de América del Norte, de Oceanía y de Europa tuvieron un baby boom caracterizado por un aumento en fertilidad (Van Bavel \& Reher, 2013), mientras los países en vías de desarrollo atravesaban la fase intermedia de su transición demográfica (Teitelbaum, 1975) con una amplia brecha entre natalidad y mortalidad. En todo caso, es erróneo asumir que las poblaciones humanas crecerán por siempre, debido a que mientras las naciones crecían demográficamente durante la segunda mitad del siglo 20, sus tasas de fertilidad disminuían (Hobbs \& Stoops, 2002) de aproximadamente 5 hijos por mujer en 1950 a 2.5 en el año 2005, y la ONU proyecta que caerá a 2 niños por mujer en el año 2050 (Bloom, Canning \& Fink, 2010). Actualmente hay 97 países y territorios (WB, 2017) que tienen tasas de fecundidad de reemplazo iguales o inferiores a 2 hijos por mujer fértil (de 15 a 45 años de edad), lo que significa que a menos que algo cambie drásticamente, toda la población mundial comenzará a disminuir a mediados del próximo siglo (DESA, 2004). De hecho, el declive de la población puede surgir de la combinación de una baja natalidad, alta mortalidad y emigración, pero en el mundo moderno, las bajas tasas de natalidad son la clave.

Este artículo se organiza de la siguiente manera: 1) el modelo de transición demográfica; 2) el fenómeno del baby boom en los países ricos del planeta a mediados del siglo 20;3) el dividendo demográfico en la cuarta fase del modelo de transición; 4) el decrecimiento poblacional que se está produciendo en algunos países y a futuro sucederá en el mundo entero; y 5) las consecuencias económicas en el Producto Interno Bruto (PIB) de este declive.

\section{EL MODELO DE TRANSICIÓN DEMOGRÁFICO}

La Revolución Industrial se inició en Inglaterra a mediados del siglo 18 (Mantoux, 2013), dándose cambios paulatinos en los procesos productivos, pasando de confeccionarse bienes con herramientas en talleres artesanales a ser producidos más eficientemente con máquinas en fábricas. Las nuevas tecnologías se difundieron a inicios del siglo 19 a los países de Europa Occidental (Newbigin, 1918) y a los Estados Unidos (McCormick, 2014), llegando a Japón en 1870 (Macpherson, 1994). Esta revolución cambió al mundo, puesto que fue acompañada de grandes avances científicos, tecnológicos (Musson \& Robinson, 1969), de infraestructura y de comunicaciones (Canning, 1998), en continuo desarrollo, originando además un aumento poblacional sin precedentes históricos. Sin embargo, este acelerado incremento no tiende a un crecimiento numéricamente infinito, sino que es una tendencia que se va modificando a través del tiempo, llegando a un momento en el cual el volumen poblacional de un determinado país se estabiliza y luego incluso disminuye. Al conjunto de estos cambios producidos en el comportamiento de las tasas de natalidad y de mortalidad se le denomina modelo de transición demográfico (Loschky \& Wilcox, 1974; Thompson, 1929; Landry, 1934; Prais, Leibenstein \& Notestein, 1955), y consta de cinco fases (Chesnais, 1992). 


\subsection{Fase 1 o preindustrial}

Se caracteriza por presentar índices de natalidad y de mortalidad altos y fluctuantes (alrededor de 40 nacimientos y muertes por cada mil habitantes), razón por la cual el crecimiento poblacional varía entre $1 \%$ y $-1 \%$ anual, decrecimiento que podía en ocasiones ser más alto debido a mortalidades originadas por hambrunas, epidemias o guerras. La pirámide poblacional típica de esta fase presenta una base ancha (predominio de población joven) con relación a su cúspide (población vieja), además sus lados son cóncavos.

\section{2. $\quad$ Fase 2}

En este período la natalidad aumenta a alrededor de 40 nacimientos por cada mil personas, en tanto que la mortalidad decrece hasta cerca de 15 fallecimientos por cada mil personas. Esto, debido a que existen mejores condiciones de vida, ya que se ha iniciado la industrialización, ocasionando un crecimiento poblacional moderado (alrededor del 2.5\% anual), el mismo que tiende a aumentar. La pirámide poblacional en este período es similar a la de la fase preindustrial, aunque con los lados rectos.

\subsection{Fase 30 intermedia}

Se caracteriza porque la natalidad empieza a descender (de 40 a 25 nacimientos por cada mil personas), en tanto que la mortalidad continúa disminuyendo (alrededor de 10 muertes por cada 1000 individuos). Ambas tasas disminuyen debido al acelerado proceso de urbanización, al aumento de los niveles de educación, y a los grandes avances en la salud preventiva (incluyendo métodos anticonceptivos) así como en la medicina curativa. En esta fase se presenta la brecha más acusada entre natalidad y mortalidad, produciéndose un crecimiento vegetativo que alcanza valores elevados (cerca de $3 \%$ anual, dependiendo del país que se trate, así los países más desarrollados o ex-socialistas presentaron en esta fase un crecimiento poblacional mucho menor con relación a los países subdesarrollados que se encuentran hoy en este período demográfico). La pirámide poblacional presenta una base ancha, con cúspide delgada (poca población vieja) y los lados de la pirámide son convexos.

\section{4. $\quad$ Fase 4}

Aquí la tasa de natalidad continúa disminuyendo (cerca de 10 nacimientos por cada mil personas al finalizar este período), en tanto que la mortalidad se mantiene baja y con valores similares a la natalidad (casi 10 muertes por cada mil individuos). El crecimiento poblacional se desacelera (menor a 1\% anual), el mismo que sigue disminuyendo. La pirámide poblacional en esta fase presenta una base menos ancha que en los períodos anteriores, mientras que la parte central corresponde a la zona más abultada, y la cúspide aumenta su espesor, en tanto que ambos lados de la pirámide son cóncavos desde su base hasta el centro, y convexos desde aquí hasta la cúspide.

\subsection{Fase 5}

Presenta una natalidad baja (menor a 10 nacimientos por cada mil habitantes), en tanto que la mortalidad aumenta por el envejecimiento poblacional hasta llegar a casi 15 muertes por cada mil individuos; razón por la cual el crecimiento demográfico es negativo (alrededor de $-0.5 \%$ anual) al finalizar este periodo. La pirámide poblacional presenta una base angosta (poca población joven), su parte central es bastante ancha (predominio de población adulta), su cúspide es de un espesor similar a su base y sus dos lados son cóncavos desde la base hasta el centro y convexos desde aquí hasta la cúspide.

El incremento poblacional según el modelo de transición demográfica puede ser descrito gráficamente mediante una curva de crecimiento logístico (Horelick, Koont \& Gootlieb, 1983) con varios puntos de inflexión, iniciándose con un crecimiento poblacional lento (fases pre-industrial e inicial), el mismo que paulatinamente se va acelerando a través del tiempo (fase intermedia), hasta llegar finalmente a desacelerarse nuevamente (en sus dos fases finales), para luego estancarse y finalmente decrecer (Fig. 1). 


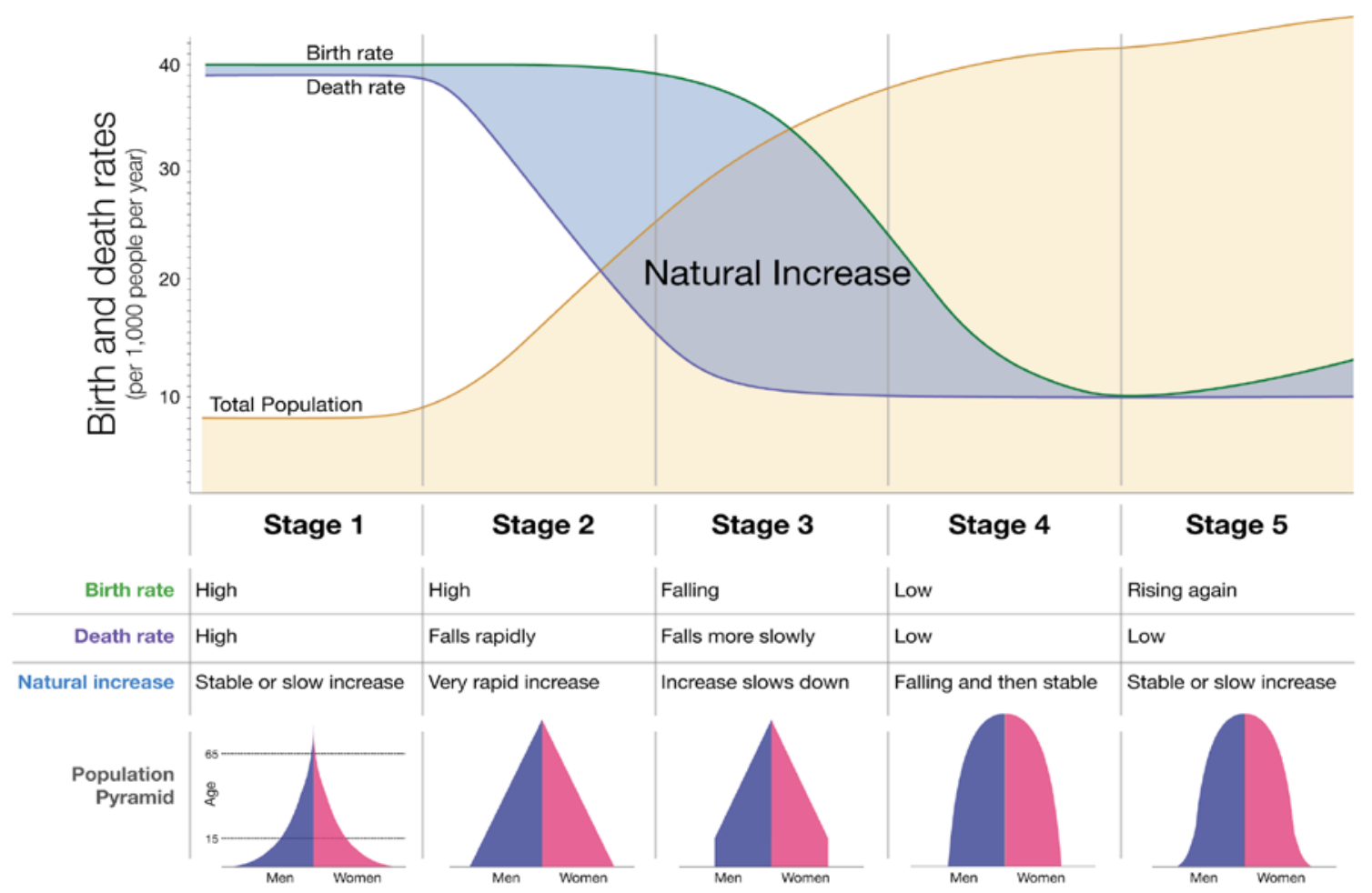

Figura 1. El modelo de transición demográfica (Roser, 2016a).

Este modelo de transición demográfica difiere entre países, encontrándose actualmente distintas regiones y naciones del planeta en varias fases de esta transición, así mientras Europa se encuentran en una fase evolucionada (Goldstein, Lutz \& Scherbov, 2003), incluso muchos de sus países del Este (Lituania, Letonia, Estonia, Rumania, Bulgaria, Hungría y Ucrania) presentan tasas negativas de crecimiento poblacional; en cambio generalmente el África Subsahariana se encuentra en la fase intermedia de esta transición, en pleno crecimiento explosivo (Faruqee \& Gulhati, 1983), presentando tasas de incremento demográfico superiores al 3\% anual (Sudán del Sur, Níger, Burundi, Chad, Angola, Uganda, Gambia, República Democrática del Congo, Nigeria, Tanzania, Senegal, Malawi y Zambia).

\section{EL BABY BOOM EN LOS PAÍSES INDUSTRIALIZADOS A MEDIADOS DEL SIGLO 20}

Durante las últimas décadas del siglo 19 y primeras del siglo 20, las tasas de natalidad empezaron a disminuir gradualmente en los países más urbanizados del planeta, excepto por el rango comprendido entre los años 1940 hasta fines de los años 1960, cuando la dinámica de reproducción cambió, con un gran número de matrimonios de parejas jóvenes que aumentaron la fecundidad en los Estados Unidos, así como en los países de Europa y de Oceanía (Van Bavel \& Reher, 2013), afectando en menor medida este fenómeno también a Japón. Las reformas económicas del Keynesianismo en la década de los 1930s (Hall, 1989), las políticas de HomeFront de los años 1940s (Harrison, 2000), la recuperación post-bélica gracias al Plan Marshall (Hogan, 1987) y a la ayuda monetaria a Japón (Yamamura, 1967), hicieron posible la reconstrucción económica de estos países, y la nueva reindustrialización generó tasas de desempleo bajas para los cohortes nacidos en los 1920s y 1930s, sintiendo la gente bienestar material y optimismo sobre el futuro, así que tenían más hijos (jamás tantos niños como las grandes familias anteriores al siglo 20), basados en "sus mejores ingresos relativos" en relación a sus aspiraciones previas y a los hogares donde fueron criados (Easterlin, 1976). En los países de Europa del Este el baby boom se dio con menor fuerza, excepto en Checoslovaquia, mientras que en Rusia y otras repúblicas de la ex Unión Soviética, las tasas de natalidad siguieron descendiendo hasta mediados de los años 1960s, cuando se mantuvieron constantes o se incrementaron ligeramente (Lutz, Scherbov \& Volkov, 2002). 
Siendo la causa principal para este incremento en los países ex-socialistas el sentimiento de repoblación luego de tantas víctimas bélicas (Fig. 2). En todos estos países el descenso de la natalidad se dio desde inicios de los años 1970 en adelante, declive que continúa actualmente.

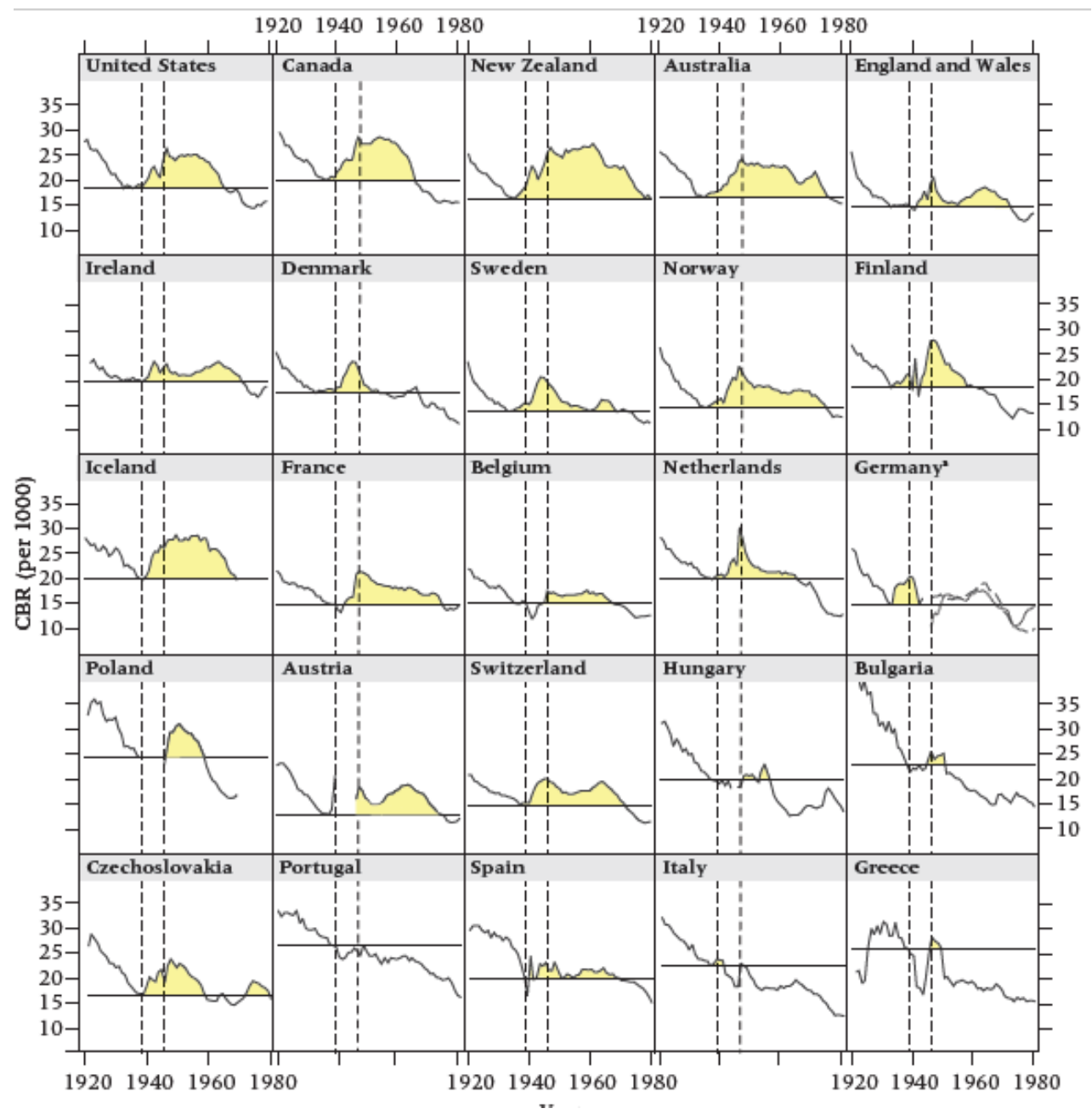

Figura 2. El baby boom en los países de Norte América, Europa y Oceanía: tasas de natalidad (Van Bavel \& Reher, 2013).

\section{EL DIVIDENDO DEMOGRÁFICO}

La palabra dividendo significa: "retorno de la inversión de capital en una corporación, la distribución de dividendos es una transacción en la que los beneficios empresariales se distribuyen a los dueños de la corporación” (Helminen, 1999). Por ende, este término implica beneficios y en demografía se refiere a las consecuencias positivas que se generan para la sociedad el hecho de que exista un alto porcentaje de población adulta y económicamente independiente en relación al porcentaje de población dependiente conformada por jóvenes y viejos (Bloom, Canning \& Sevilla, 2003). Esto sucede cuando los países se encuentran atravesando la fase 4 del modelo de transición demográfica, puesto que la fase 3 de este modelo se caracteriza por presentar muchos niños, mientras que los hogares y gobiernos destinan sus esfuerzos y recursos económicos para su cuidado, alimentación, vestimenta, salud y educación. En cambio en la fase 5 de este modelo, las familias y los estados tienen un alto porcentaje de viejos y por ende destinan ingentes cantidades de dinero para sus pensiones, cuidado y salud (Mason, 2005). 
Sin embargo, el dividendo demográfico implica únicamente una posibilidad, una supuesta ventana de oportunidad; el término fue aplicable anteriormente para los países más ricos del planeta, pero en la práctica es inaplicable para muchas naciones que se encuentran actualmente en esta cuarta fase, ya que muchas de ellas presentan altas tasas de subempleo y de desempleo en relación a su población económicamente activa, razón por la cual no siempre se puede reclamar los beneficios positivos que este dividendo debería traer a las sociedades. Brasil (Turra \& Queiroz, 2005), Argentina (Gragnolati, Rofman, Apella \& Troiano, 2015), México (Partida-Bush, 2004) y muchos estados de América Latina se encuentran hoy en su dividendo demográfico desde el punto de vista de sus estructuras poblacionales con un bajo nivel de dependencia; sin embargo, en la práctica y desde la visión económica tienen elevados niveles de dependencia y de pobreza debido a problemas relacionados con la falta de oportunidades laborales (Cruces, Fields, Viollaz \& Jaume, 2017).

\section{DECRECIMIENTO POBLACIONAL EN PAÍSES QUE SE ENCUENTRAN AL FINAL DE SU TRANSICIÓN DEMOGRÁFICA}

En base a series históricas de la Organización de las Naciones Unidas (ONU) y otros autores, el primer país en presentar tasas de fecundidad de 2 hijos por mujer, o menos, fue Letonia en dos períodos: desde 1950 hasta 1982 (Katus, 1997) y desde 1991 hasta la actualidad (WB, 2017). De manera similar Luxemburgo presentó estas tasas desde 1950 hasta 1955 (DESA, 2015) y desde 1970 (Mauldin, 1976) hasta la actualidad (WB, 2017). Luego, Estonia empezó a tener sub-fertilidad de reemplazo desde 1955 hasta 1967 (Katus, 1991) y desde 1991 hasta la actualidad (WB, 2017); posteriormente Hungría presentó este fenómeno en tres ocasiones: desde 1961 a 1966, de 1970 a 1973, y de 1980 hasta la actualidad (Spéder \& Kamarás, 2008). Con el transcurrir del tiempo, otros países siguieron esta tendencia, de tal manera que en el año 1975 hubo 20 países y territorios no independientes que presentaban tasas de fertilidad inferiores a la fecundidad de reemplazo. Este número se incrementó a 76 en el año 2000 y a 97 en el año 2015 (WB, 2017), de un total 254 países y territorios en existencia (DESA, 2015) (Fig. 3). A pesar de que la población no ha dejado de crecer en los últimos 50 años en el mundo, el promedio de hijos por mujer ha caído aproximadamente en un $50 \%$, afectando actualmente la sub-fertilidad a casi el 50\% de las naciones, un patrón que persistirá en el futuro. Dentro de este grupo se encuentran países de Europa, (e.g. Rusia, Alemania, Italia, Reino Unido, etc.) Asia del Este (e.g. China, Japón, Corea del Sur, etc.), Norte América (EE.UU. y Canadá), Oceanía (Australia y Nueva Zelanda) y América Latina (e.g. Brasil, Colombia, Chile, etc.) (WB, 2017), estimándose que ascenderá a 116 naciones para el periodo 2025-2030, a 153 entre 2050-2055, a 178 entre 2075-2080 y a 198 estados para 2095-2100 (DESA, 2015), implicando que en el futuro el detrimento poblacional afectará a la mayoría de países (excepto a aquellos que tienen altos saldos migratorios positivos).

Si bien es cierto, la población mundial sigue aumentando hoy, está creciendo más lentamente a medida que transcurren los años. La proyección (hipótesis media) de la ONU calcula que el planeta llegará a tener 9,725 millones de individuos en el año 2050, y a 11,213 millones en el 2100 (DESA, 2015), sin estabilizarse durante este siglo (Gerland, Raftery, Sevčiková, Li, Gu et al., 2014), y llegando a su pico máximo a mediados del próximo siglo (DESA, 2004). Además la variante media de las proyecciones de la ONU supone sin evidencia científica que todos los países convergerán eventualmente a un equilibrio, con una tasa global de fecundidad de 1.85 hijos (DESA, 2013). Con una mortalidad constante y sin migración, dicha tasa implica un eventual descenso en la población de alrededor del 0.35\% anual. Las otras dos proyecciones (hipótesis alta y baja) también elaboradas por la ONU son menos factibles de concretarse en la realidad (Keilman, 1998; Keyfitz, 1981) (Fig. 4). 

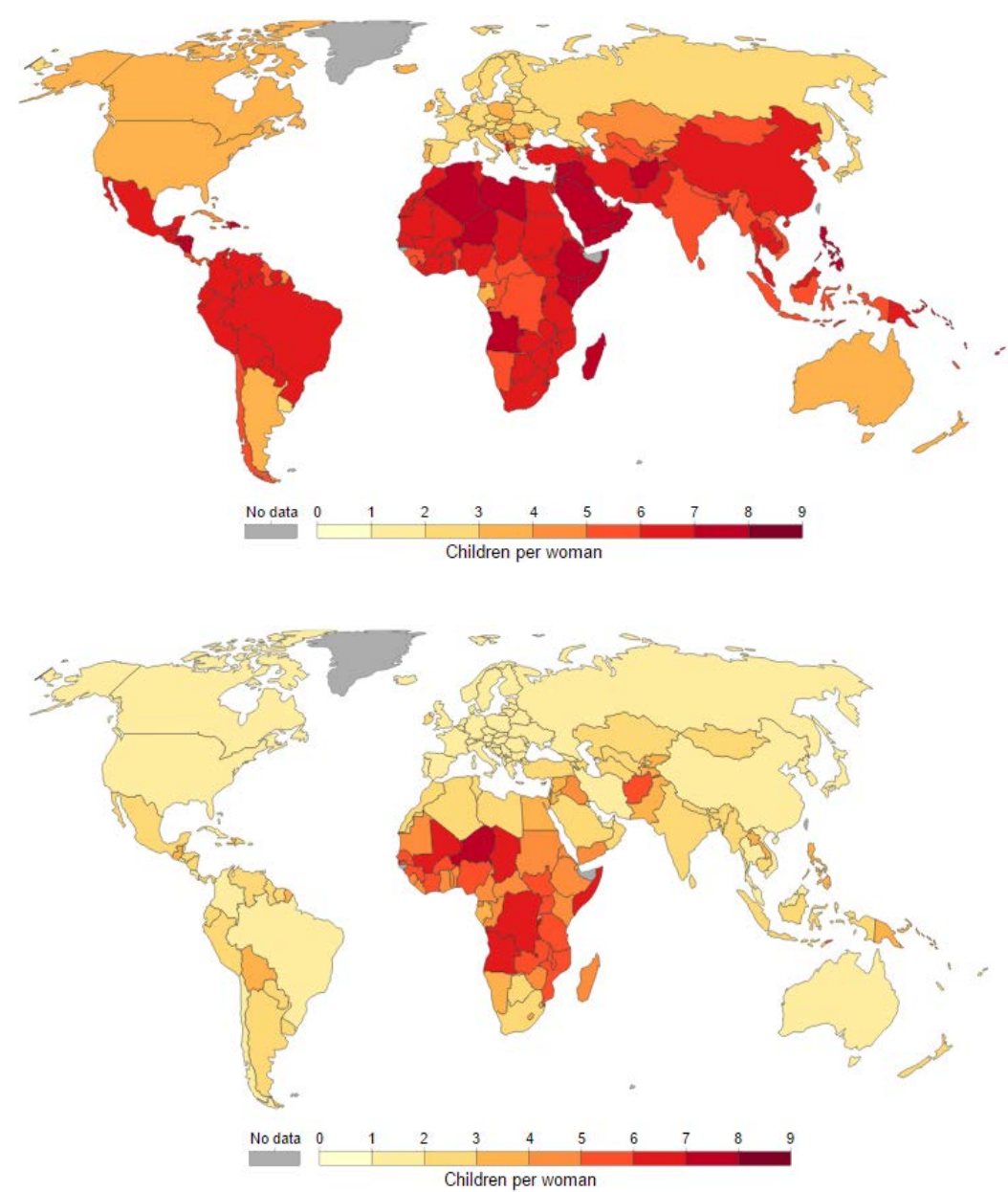

Figura 3. Tasas de fertilidad en los diferentes países y territorios en 1955 (mapa superior) y 2015 (mapa inferior) (Roser, 2016b).

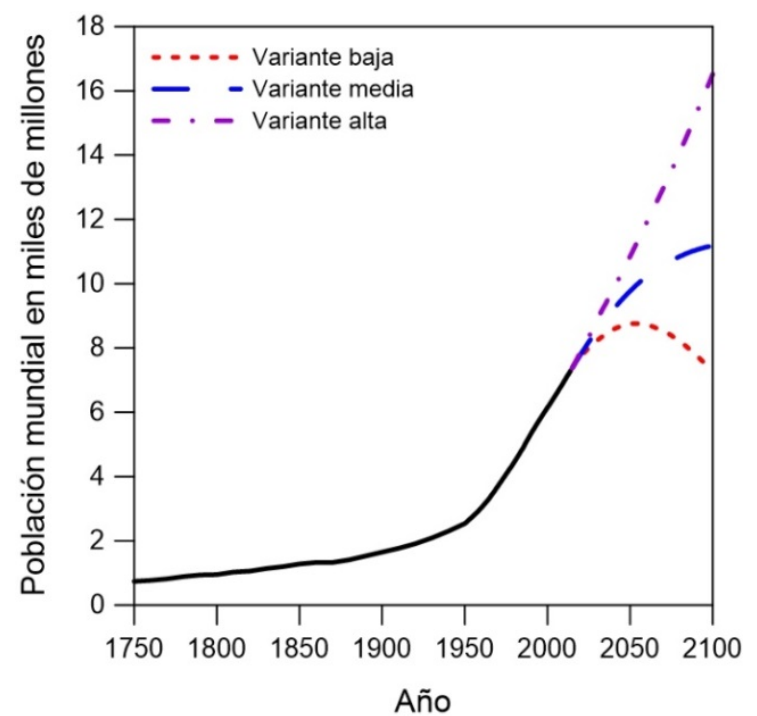

Figura 4. Estimaciones y previsiones de la población mundial por la ONU, 1950-2100 (Roser, 2016c). Nota: La variante media es la proyección que los investigadores de la ONU consideran el escenario más probable. La variante alta y baja simplemente suponen que las tasas de fecundidad total en cada país son 0.5 más altas y 0.5 más bajas que la variante media para fines de este siglo en cada país. 


\section{CONSECUENCIAS ECONÓMICAS EN EL PIB DEL DECRECIMIENTO POBLACIONAL}

Los argumentos económicos a favor del crecimiento demográfico se basan en la reducción de los mercados y su efecto deprimente sobre la inversión-innovación (Coleman \& Rowthorn, 2011), refiriéndose a la infraestructura empresarial, donde el stock de capital tiene una larga vida útil, y si la población crece rápidamente, las empresas pueden construir adelantándose a la demanda, por lo que la capacidad instalada se infra-utiliza inicialmente, pero se va utilizando más y más, a medida que aumenta la población, razón por la cual la perspectiva de crecimiento futuro hace que los inversores sean más aventureros a la espera de ventas mayores. Pero, cuando la población y demanda se contraen secuencialmente, sucede todo lo contrario, ya que la utilización de la capacidad instalada disminuye a medida que cae el número de usuarios, por ende, los inversores se mostrarán reacios a adquirir maquinarias de mayor productividad y producción porque les preocupa el exceso de su capacidad instalada futura.

Estos argumentos dependen del horizonte temporal del producto en cuestión y del ritmo de disminución de la población, así para productos con un ciclo de vida muy corto, menores a cinco años, hay poca diferencia entre un mercado que se expande al $0.5 \%$ anual y uno que se está reduciendo a este mismo ritmo, ya que después de cinco años, el mercado en expansión es apenas un $5 \%$ más grande que el mercado en contracción y tal diferencia no tendría mayor efecto sobre el incentivo para invertir. Pero, en un período de 50 años, que es el horizonte temporal que se aplica a la vivienda y muchos proyectos de infraestructura, se generará una divergencia sustancial entre un mercado que crece y uno que está en decadencia, ya que luego de transcurrido este medio siglo, el mercado en expansión será un $50 \%$ más grande que el mercado en retroceso (Coleman \& Rowthorn, 2011).

De acuerdo a la visión más convencional, el final del crecimiento demográfico causado por la disminución en las tasas de fertilidad, conduce a una población de mayor edad y a un menor número de trabajadores, consecuentemente se generan tasas de dependencia de la vejez cada vez más altas (Lee \& Mason, 2010) y cambios en las pirámides poblacionales que plantean una preocupación válida para los gobiernos sobre la posible desaceleración del crecimiento económico (Bloom et al., 2010). Debido a que la mano de obra es esencial para que existan procesos productivos, y es obvio que el crecimiento de la población aumenta la producción total (PIB), siempre que se puedan emplear trabajadores adicionales. Por el contrario, el detrimento demográfico implica una disminución, estancamiento o crecimiento más lento de la producción, a menos que éste sea compensado por la aceleración de la productividad. Por ende las economías de escala pueden disminuir, generándose una menor demanda interna, una contracción de los mercados y menor rentabilidad empresarial. Además, la perspectiva de mercados en declive influye en inversiones más débiles, lo que significaría el futuro envejecimiento de las máquinas y menos competitividad, de hecho la vulnerabilidad puede ser mayor si no existe perspectiva de crecimiento a largo plazo de la demanda agregada para aumentar la confianza, pudiendo empezar a existir importaciones más baratas que reemplacen a la oferta interna, hundiéndose por ende la capacidad de producción nacional (Coleman \& Rowthorn, 2011). Además, el envejecimiento de las personas conlleva a la reducción gradual de la capacidad productiva, a medida que la fuerza de trabajo más vieja cae no solo en número sino también en su capacidad de producción e innovación (Lindh, 2005; Skirbekk, 2005). Por ejemplo, las industrias de servicios requieren nuevas habilidades, especialmente en el campo de las tecnologías de la información, y las personas mayores son generalmente refractarias a las mismas.

Para que no exista disminución en el tamaño de las economías modernas, o en otras palabras, para evitar el decrecimiento del PIB hay dos alternativas: 1) mantener o aumentar la Población Económicamente Activa (PEA) de un país, ya que al disminuir el número de personas, no habrá suficiente gente para producir los bienes y servicios que se necesitan, pudiéndose generar un doble colapso, tanto desde el punto de vista de la producción como del consumo (Coleman \& Rowthorn, 2011), o 2) como piensa Gary Becker (Premio Nobel en Ciencias Económicas en 1992) y otros, esto es, aumentar la productividad de la PEA de una nación así como el consumo individual para mantener el nivel deseado de inversión y confianza, y, de hecho, la baja fecundidad impulsa el envejecimiento de la población. Pero también está asociada al aumento de las tasas de inversión en el capital humano de 
niños y jóvenes, de modo que cada uno de los pocos trabajadores tiende a ser más productivos, compensando así los efectos del aumento de la tasa de dependencia de la vejez gracias a aumentos paulatinos en la productividad de los trabajadores (Lee \& Mason, 2010). En otras palabras a través de invertir en habilidades, conocimiento e información que la gente adquiere para transformar a través de la investigación científica y de la creación de nuevas tecnologías a las economías modernas, volviéndolas más productivas (Becker, Murphy \& Tamura, 1990). Los países que no son capaces de mantener sustanciales inversiones para potenciar el intelecto humano de sus pueblos no serán capaces de convertirse en un economías modernas o desarrolladas (Becker, 2002). Los aumentos en la productividad son de vital importancia para el crecimiento de las economías y para reducir los costos de producción y por ende generar más poder adquisitivo. Entonces, si la población en edad de trabajar se redujera y los países tuvieran cada vez menos trabajadores, cada uno de ellos deberá ser más productivo, ya sea trabajando más duro o más inteligentemente para mantener los niveles de producción anteriormente alcanzados; es decir, que el declive demográfico se transforma en un reto mucho mayor sólo para mantener los niveles actuales de vida (Fig. 5).

El reto de los gobiernos de muchas naciones consistirá en aumentar los niveles de educación de su población (Becker \& Chiswick, 1966), ya que, con altos niveles de conocimientos, las sociedades generarán más y más innovaciones tanto científicas como tecnológicas. De manera similar, Julian Simon y Albert Bartlett (1985) en su libro: 'The Ultimate Resource” dicen que el factor clave en el crecimiento económico consiste en la capacidad para crear nuevas ideas que contribuyan al avance del conocimiento. Por ende, no es necesariamente el aumento poblacional, sino la capacitación de las personas lo que contribuye a resolver los diferentes problemas, eliminándose más rápido los obstáculos y generándose una mayor herencia económica para las futuras generaciones.

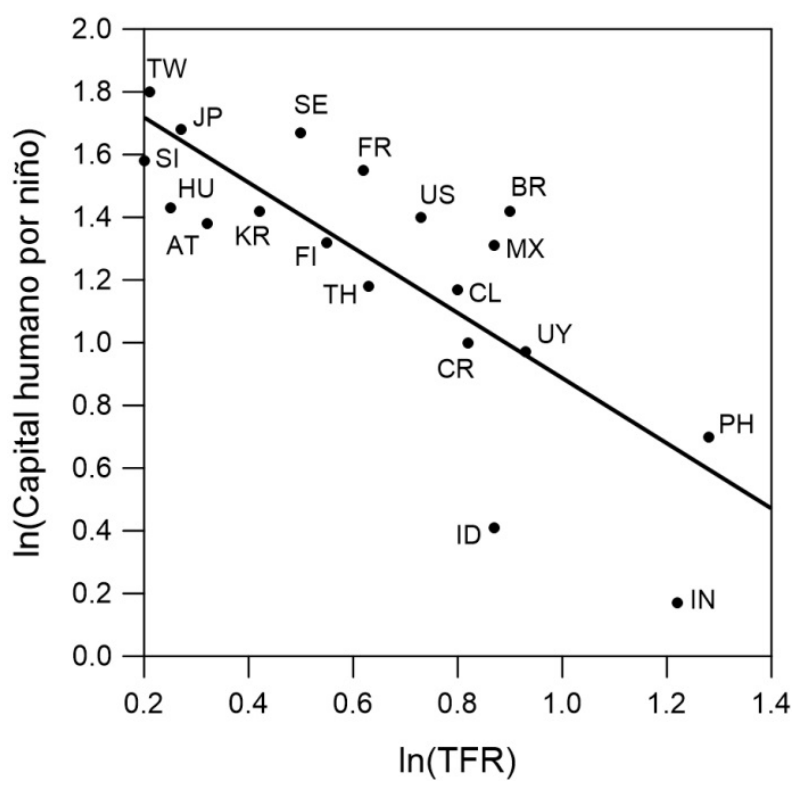

Figura 5. Gasto de capital humano por niño (público y privado) versus la tasa de fecundidad total (Lee \& Mason, 2010; Ogawa, Mason, Chawla, Matsukura \& Tung, 2009). Nota: El gasto de capital humano se normaliza dividiéndolo por el ingreso laboral promedio de los adultos de 30 a 49 años (Lee \& Mason, 2010). [AT = Austria; BR = Brasil; $\mathrm{CL}=$ Chile; $\mathrm{CR}=$ Costa Rica; FI = Finlandia; FR = Francia; $\mathrm{HU}=$ Hungría; ID = Indonesia; IN = India; JP = Japón; KR = Corea del Sur; MX = México; PH = Filipinas; SE = Suecia; SI = Eslovenia; TH = Tailandia; TW = Taiwán; US = Estados Unidos; UY = Uruguay .

Existen algunos estudios basados en la asociación estadística de las proporciones de población joven y anciana con el crecimiento económico, seis de estos estudios se exponen a continuación:

1) Huh, Lee \& Lee (2007) utiliza un modelo de ajuste parcial con datos de 77 países durante 2 años, sus resultados indican que una población relativamente grande de niños y ancianos tiene un efecto negativo sobre el crecimiento económico, encontrándose que el tamaño relativo de los ancianos 
en la población total tiene un mayor efecto negativo en el crecimiento económico que un aumento en el número relativo de niños.

2) Por el contrario, Bloom, Canning \& Fink (2010) basados en una sistemática recolección de datos demuestran en un modelo que la tasa de envejecimiento afecta negativamente al crecimiento económico en el corto plazo e insignificantemente en el largo plazo. Lo que sucede es que el efecto negativo del envejecimiento es mitigado por una participación más activa de la mano de obra de mayores de 65 años y más ahorros de jubilación, llegando a calcular que los países de la Organización para la Cooperación y el Desarrollo Económico (OCDE) probablemente experimenten una disminución modesta, pero no catastrófica, de la tasa de crecimiento económico. Mientras que en la mayoría de naciones no pertenecientes a la OCDE, la disminución de las tasas de fecundidad generará que la proporción de la fuerza de trabajo aumente y los niveles de dependencia disminuyan gracias al dividendo demográfico, sugiriendo por ende que el envejecimiento de la población no obstaculizará significativamente el ritmo de crecimiento económico de los países en desarrollo.

3) Prettner (2013) en un estudio sobre crecimiento económico endógeno demuestra que los aumentos en la longevidad tienen un impacto positivo en el crecimiento de la producción per cápita, mientras la disminución de la fecundidad tiene un impacto negativo en el crecimiento de la producción per cápita, pero al final el efecto positivo de la longevidad domina sobre el efecto negativo de la fertilidad.

4) Jones (1995) utiliza un modelo de crecimiento semi-endógeno, y demuestra que los siguientes escenarios pueden suscitarse: (i) si la mortalidad disminuye a un ritmo menor que la fecundidad, el envejecimiento de la población afecta negativamente al crecimiento económico a largo plazo; (ii) si la mortalidad disminuye de manera similar a la tasa de disminución de la fecundidad, el envejecimiento de la población no tiene efecto en el crecimiento económico a largo plazo; y (iii) si la mortalidad disminuye a un ritmo más elevado que la fecundidad, el envejecimiento de la población repercute positivamente en el crecimiento económico a largo plazo.

5) Kaldor (1996) manifiesta que el crecimiento de la productividad está relacionado directamente con el incremento de la producción, especialmente en el sector manufacturero, por ende en una economía de rápido crecimiento, las empresas innovan y mejoran la eficiencia a un ritmo más rápido porque deben superar constantemente nuevos desafíos, pero si la disminución de la población conduce a generar menos innovación, se llegará a estancamientos productivos y declives en la producción.

6) Matsutani (2006) dice que una disminución en la tasa de crecimiento de la población tendrá un impacto en la estructura productiva de la economía nacional, ya que la demanda interna de bienes de capital (máquinas), las edificaciones y la infraestructura dependen de la tasa esperada de incremento de la producción; por lo tanto, si la disminución de la población conduce a un crecimiento más lento, estancamiento o disminución de la producción, es probable que el resultado sea un cambio en la composición de la demanda interna, destinándose menos dinero para adquirir bienes de capital, mientras que aumentará el consumo de bienes y servicios. Las implicaciones para los productores de maquinarias dependerán de la velocidad con la que se produzca la transición de una inversión interna alta a baja y de la importancia de las exportaciones en la economía. Al generarse esta transición de manera gradual, las empresas que producen bienes de inversión pueden expandir sus exportaciones para compensar la pérdida de sus mercados nacionales; alternativamente, pueden reducir su producción de bienes de inversión de manera ordenada para que otros tipos de productos puedan tomar el relevo.

Irlanda fue un ejemplo único de disminución de la población debido a masivas emigraciones desde la década de los años 1840 hasta la década de los 1950, aunque solo fue un estado-nación desde 1922 (Daly, 2006; Mokyr \& Grada, 1984). Los informes oficiales manifiestan la existencia de altos costos en la provisión de servicios, un limitado mercado interno, el desaliento para asumir riesgos y la falta de optimismo sobre las perspectivas futuras (Berelson, 1974). En la actualidad muchos países de Europa del Este como Letonia, Bulgaria, Serbia y Ucrania están experimentando una disminución anual de la 
población cercana al $0.5 \%$, sufriendo combinaciones severas de baja fertilidad, alta mortalidad y emigración heredadas de la historia reciente (Coale, 2017).

En la República Federal Alemana, las cifras poblacionales disminuyeron ligeramente de 1973 a 1985, a pesar de ello, el PIB alemán siguió creciendo sustancialmente, en un $26 \%$ en comparación con el 29\% en 13 países de Europa Occidental (UNECE, 2002), sin producirse una crisis de confianza empresarial. En Japón el pesimismo económico tampoco es universal (Chapple, 2004), ya que durante la década de 1995 al 2005, el PIB japonés aumentó en un 12\% mientras que la población apenas creció en un 1.8\% (Coulmas, 2007; Matsutani, 2006), mientras que entre los años 2005 y 2015, la población disminuyó en un 1.2\%, pero el PIB japonés aumentó en un 10.6\% adicional (Chiavacci \& Hommerich, 2016). En estos dos países: Alemania y Japón, su decrecimiento demográfico se encuentra en la actualidad en torno al $-0.2 \%$ por año y de continuar esta tendencia implicaría una reducción del $10 \%$ de sus poblaciones cada 50 años (Bacci, 2017). Sin embargo, se proyecta que las alteraciones en la estructura de edades aceleren el ritmo de la población y cambien la tendencia promedio hasta mediados del siglo 21 aproximadamente a $-0.6 \%$ en Alemania y a $-0.8 \%$ en Japón, implicando una reducción de la población igual al 26\% y 33\% respectivamente en ambos países en un periodo de 50 años (Jorgenson, \& Vu, 2017). Bajo estas condiciones, la participación de la inversión en el PIB será seguramente menor debido a que se requerirá una menor capacidad productiva para satisfacer la demanda futura (Poterba, 2016).

Varios países europeos (Austria, Reino Unido, Alemania, Suecia) han perdido territorio y por ende su población disminuyó en el último siglo, sin consecuencias adversas para el nivel de vida individual (Coleman \& Rowthorn, 2011). Si bien un gran mercado interno es obviamente una ventaja, como lo demuestra los Estados Unidos (Pe'er, Vertinsky \& Keil, 2016). Una ventaja equivalente también puede surgir de la adopción de la membresía en un bloque comercial como el mercado único europeo (Borner \& Grubel, 2016), pudiendo incluso las exportaciones compensar la contracción de los mercados nacionales.

Los primeros estudios de correlación entre el crecimiento demográfico y el crecimiento económico fueron realizados en 1967 por Kuznets (1967) y Easterlin (1967), economistas inminentes en las áreas de historia de las estadísticas y en demografía. Ambos autores creían que los datos que presentaban eran lo suficientemente relevantes para el tema en cuestión, y eran lo suficientemente significativos. Sin embargo, muchos autores desconocen estas correlaciones, así por ejemplo Lee en 1983 manifiesta que: "decenas de estudios, comenzando con Kuznets, no han encontrado ninguna asociación entre la tasa de crecimiento de la población y la tasa de crecimiento del ingreso per cápita, a pesar del hecho obvio de que al menos desde la Segunda Guerra Mundial, las tasas de crecimiento de la población han variado considerablemente. Estos estudios controlan otros factores como el comercio, la ayuda y la inversión en diversos grados. A nivel de países, dos estudios recientes agregan profundidad histórica a este análisis (enfocados a la existencia de desequilibrios) y, durante períodos de hasta un siglo o tan cortos como 25 años, "no existe una asociación significativa de la tasa de crecimiento de la población y la tasa de cambio del ingreso per cápita para países desarrollados o menos desarrollados" (Lee, 1983). Repetto en 1984 también afirma que: "No importa cuántas veces se repita el ejercicio, o si ha sido realizado por ganadores del Premio Nobel. Nadie ha ganado un premio por tal análisis, ni lo hará jamás. Es falacia. Cada graduado de un curso básico en economía o cualquier otra ciencia social lo sabe. Sin embargo, estas correlaciones simples continúan citándose como evidencia de que el crecimiento de la población no tiene ningún efecto en los niveles de vida" (Simon \& Kahn, 1984). El National Research Council (1986) finalmente manifestó que: "Las correlaciones simples entre el crecimiento de la población y el ingreso per cápita, aunque intrigantes, en última instancia, proporcionan poca información sobre el impacto causal de una disminución de la fecundidad impulsada por las políticas. Una evaluación científica del impacto requiere que se identifiquen los principales mecanismos mediante los cuales se supone que el crecimiento de la población afecta el desarrollo económico; evaluar la evidencia para cada hipótesis; y, finalmente, sintetizar el efecto neto de la operación simultánea de estos mecanismos” (National Research Council, 1986).

Ante estos argumentos, Simon (1989) contradice las críticas escribiendo: "Los estudios empíricos de la relación entre las tasas de desarrollo económico y de crecimiento poblacional pueden interpretarse razonablemente, sobre la base de los cánones estándares de la práctica científica, como una evidencia consistentemente sólida de la ausencia de un efecto causal negativo de la última sobre la primera, 
durante los periodos de observación. Y debido a que los períodos de observación son solo entre un cuarto de siglo y un siglo, durante los cuales los efectos parciales negativos pueden revelarse completamente, pero los efectos parciales positivos solo se revelan de manera incompleta, los estudios están sesgados en contra de la apariencia de un efecto positivo. Por lo tanto, estos estudios son consistentes con la existencia de un efecto positivo a largo plazo del crecimiento de la población sobre el desarrollo económico". Posteriormente, Barlow en 1994 realiza un estudio entre crecimiento poblacional y económico en 144 países, argumentando que: (1) en el corto plazo, un aumento en la fecundidad tiende a tener efectos negativos en el crecimiento del ingreso per cápita; (2) a la larga, sus efectos parciales tienden a ser positivos; (3) debido a que la fertilidad de hoy está altamente correlacionada con la fecundidad pasada, las tasas actuales de crecimiento de la población capturan tanto los efectos negativos a corto plazo como los positivos a largo plazo; (4) por lo tanto, en una correlación de dos variables, el crecimiento de la población actual parece tener un impacto cero en el crecimiento actual del ingreso per cápita, incluso cuando realmente tiene un efecto negativo a corto plazo (Barlow, 1994).

Finalmente, Kelley y Schmidt en 1994 realizaron correlaciones de países desarrollados y subdesarrollados para entender el impacto de la tasa de crecimiento poblacional en el crecimiento de la producción per cápita, llegando a las siguientes conclusiones: 1) No es estadísticamente significativo en la década de 1960 o 1970; 2) Es negativo, estadísticamente significativo y grande en la década de 1980; 3) Varía según el nivel de desarrollo económico: es negativo en los países subdesarrollados y a veces positivo para los países desarrollados; 4) La densidad de la población es el único indicador demográfico que ejerce un significado estadísticamente positivo en los años sesentas y setentas; y 5) Todos los componentes del cambio demográfico ejercen un efecto estadísticamente significativo en la década de 1980, donde el efecto del crecimiento de la población en el crecimiento económico es negativo y los efectos del tamaño de la población y la densidad de población son positivos (Kelley \& Schmidt, 1994).

Ciertamente, al existir disminución poblacional, el PIB tiende a crecer a un ritmo lento, a mantenerse o incluso a decrecer (Coleman \& Rowthorn, 2011). Sin embargo, lo más importante es el crecimiento del PIB per cápita (el monto que le tocaría a cada persona en un país determinado si la riqueza estuviera perfectamente bien distribuida) y del PIB per cápita PEA (el monto que le tocaría a cada persona económicamente activa en una nación si la riqueza estuviera perfectamente bien distribuida). Obviamente, excluyendo a los desempleados que también son parte de este indicador (Donoso Correa, 2000). En otras palabras, la disminución del PIB se ve confrontada con el aumento del PIB per cápita y del ingreso disponible por hogar, que son los indicadores que verdaderamente están relacionados con el bienestar individual (Sauvy, 1969), tal y como destacó la Cámara de los Lores del Reino Unido en un informe en el año 2008 (Coleman \& Rowthorn, 2011) y tal como se demuestra en el análisis económico-demográfico de cinco países que han reducido su población últimamente: Estonia, Hungría, Japón, Letonia y Hungría (Fig. 6a, b y c). De hecho, la preocupación por el PIB sólo puede justificarse si el poder nacional, la defensa y la influencia internacional tienen más peso que el bienestar de la gente para los estrategas y gobernantes, puesto que para los empleados y obreros es más conveniente la escasez de su fuerza de trabajo, ya que el precio de venta de la misma se incrementará. De hecho, actualmente la preocupación de los países que se encuentran en la última fase de su transición demográfica es la escasez de mano de obra que obstaculiza la producción y las presiones salariales inflacionarias, a diferencia de la que sucedía en la década de 1930, donde el desempleo y los recursos parados era el dolor de cabeza de las naciones más industrializadas del planeta (Keynes, 1968).

Al analizar el porcentaje de la población en edad de trabajo (de 15 a 64 años) en estos mismos cinco países, se observa que, en Japón, desde inicios del siglo 21 disminuye; mientras que en los dos países bálticos aumenta hasta el año 2006 y luego empieza a descender; en tanto que en Hungría y Polonia aumenta hasta los años 2007 y 2010 respectivamente, para luego empezar a declinar. Este declive de la población en edad productiva se debe al aumento de los cohortes en edades superiores a los 65 años de edad; sin embargo, en la práctica, las tasas reales de ocupación de la población (con respecto al porcentaje total de personas de 15 a 64 años) aumentan en todos los países en mención, con excepción de la gran recesión económica entre los años 2008 al 2012, pudiéndose apreciar una rápida recuperación de estas tasas influenciadas por dos factores: 1) una redención del PIB, y 2) el 
envejecimiento y el decrecimiento poblacional (Yenilmez, 2015), las mismas que sin lugar a dudas, colaboraron en lograr esta pronta mejoría del empleo (Fig. 7a y b).

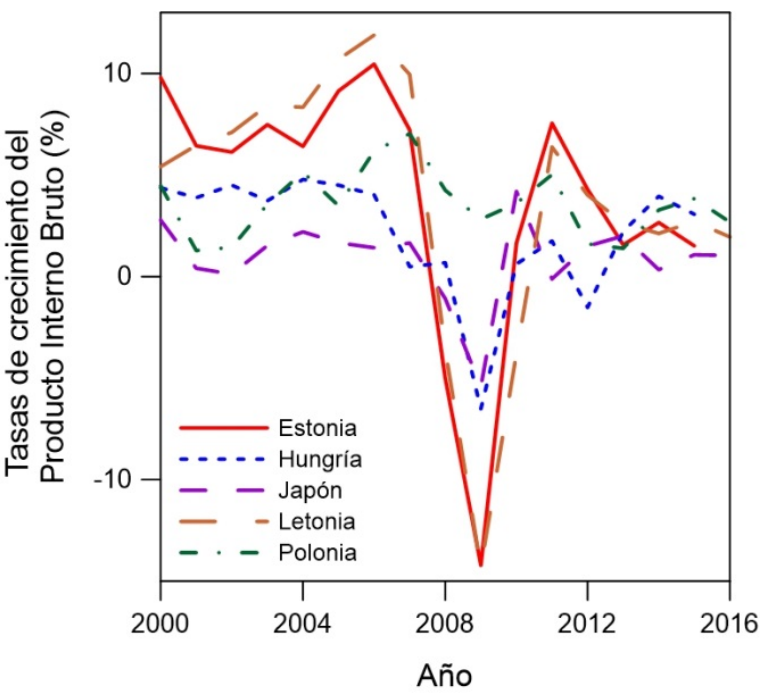

(a)

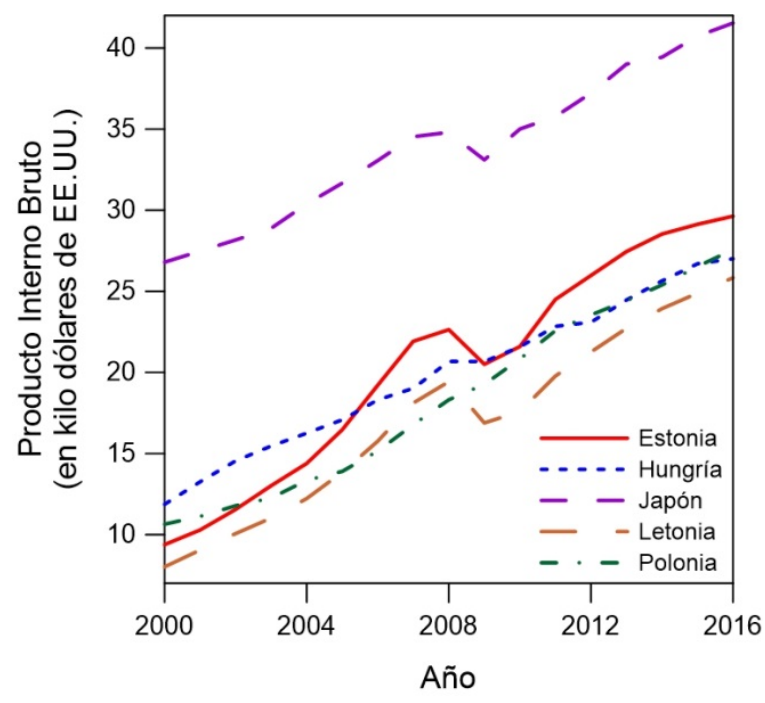

(b)

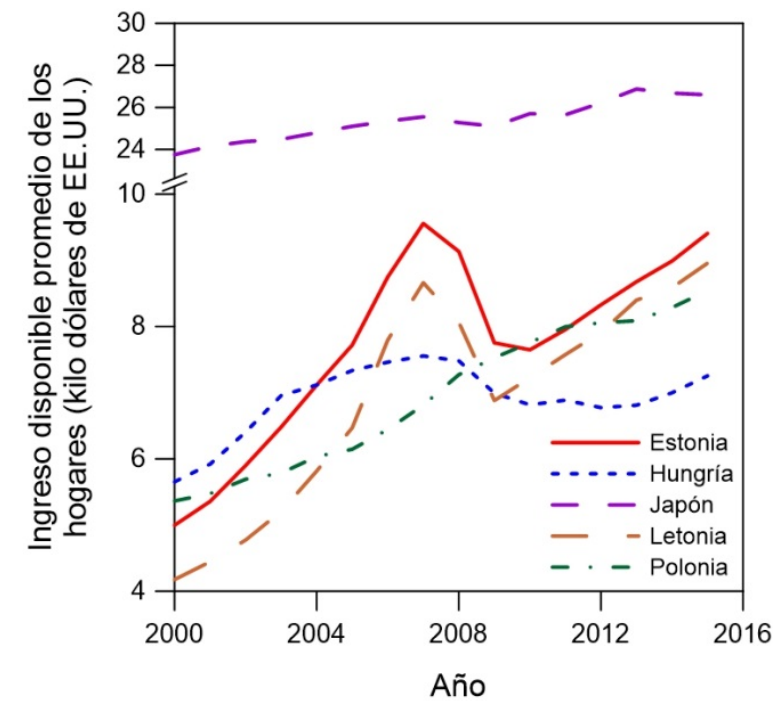

(c)

Figura 6. (a) Tasa de crecimiento del Producto Interno Bruto en porcentajes; (b) Producto Interno Bruto per cápita en kilo dólares de EE.UU.; y (c) Ingreso disponible promedio de los hogares en kilo (i.e. miles) dólares de EE.UU. (OECD data, 2017).

Por todo lo mencionado previamente, es obvio que, con excepción de Japón, las otras naciones hayan tenido altos niveles de desempleo entre los años 2008 y 2010, sin embargo, éstos pudieron descender drásticamente en poco tiempo, debido a los mismos factores mencionados anteriormente y que influyeron en el aumento de la tasa de ocupación de la población (Fig. 8).

A través del análisis de estos gráficos sobre indicadores económicos, correspondientes a cinco países que se encuentran en la fase final del modelo de transición demográfica, se aprecia claramente los efectos positivos que conlleva el envejecimiento y el decrecimiento poblacional, no tanto a escala macroeconómica, pero más bien a nivel microeconómico y de bienestar de los hogares. De hecho, y a diferencia de lo que sucede con la población joven, que es dependiente de los cohortes en edad de trabajo, el segmento de los viejos no indica necesariamente que se trate de una "carga económica" y tampoco inhibe el crecimiento económico en el corto y largo plazo. Más bien, en muchos países, las 
respuestas de comportamiento al envejecimiento de la población se traducen en una mayor participación de personas mayores a 65 años en la fuerza de trabajo y mayores ahorros de jubilación (Lee et al., 2013).

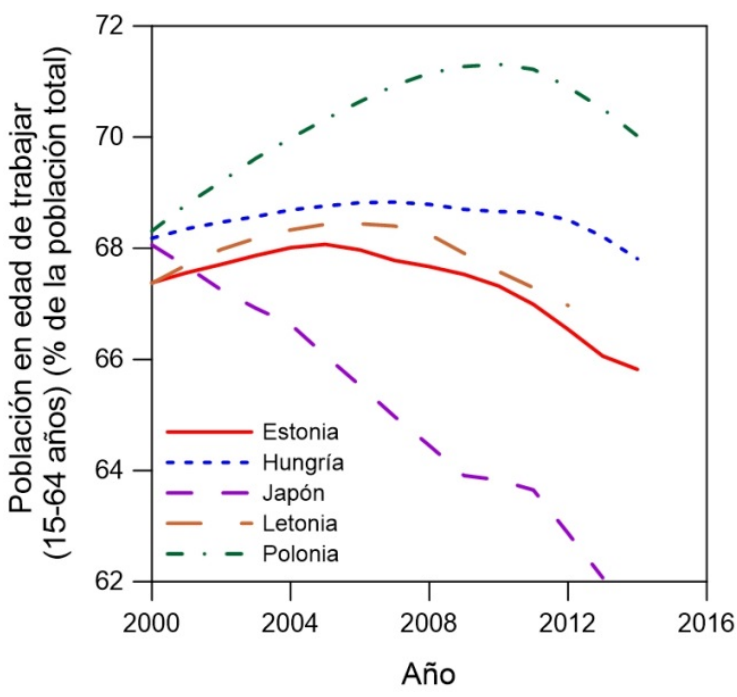

(a)

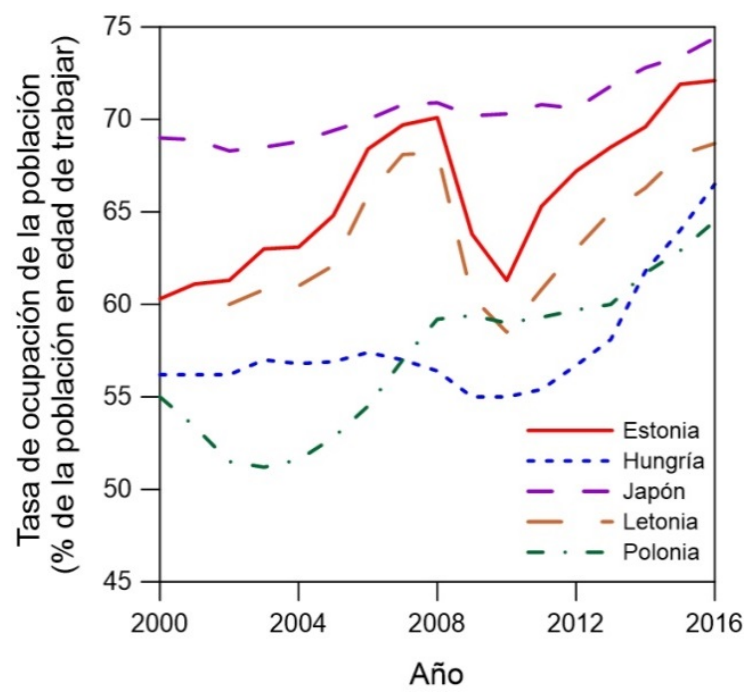

(b)

Figura 7. (a) Población en edad de trabajo de 15 a 64 años (porcentajes con respecto a la población total; y (b) Tasa de ocupación de la población (con respecto al porcentaje total de personas de 15 a 64 años) (OECD data, 2017).

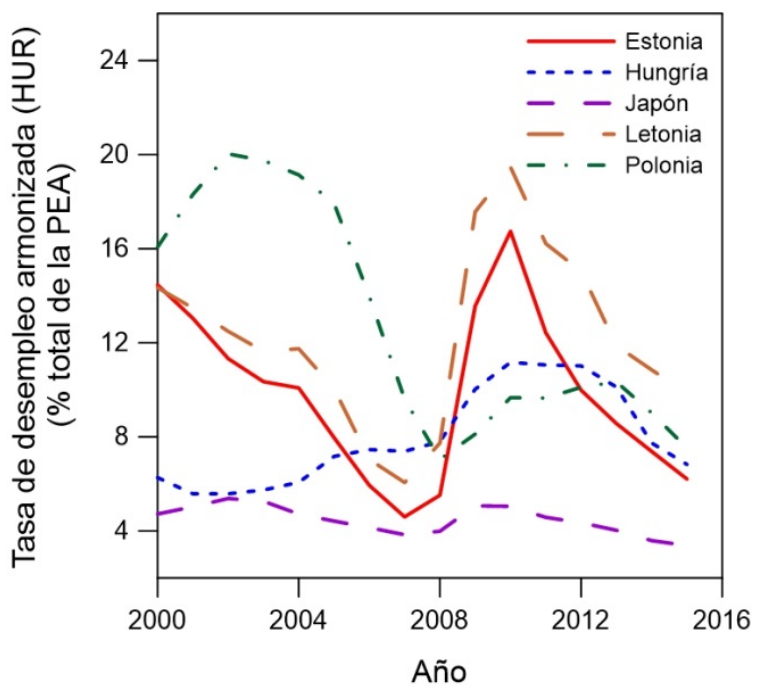

Figura 8. Tasa armonizada de desempleo o HUR (con respecto al porcentaje total de la PEA) (OECD data, 2017).

Con el transcurrir del tiempo, al disminuir aún más la población, habrá empresas que tendrán que disminuir su producción para el mercado interno, y a no ser que puedan exportar, algunas de ellas tendrán que cerrar sus puertas, prevaleciendo en el mercado aquellas que tengan más utilidad o rentabilidad neta total o porcentual (con relación a la inversión). Aquí los gobiernos tendrán que intervenir subsidiando o disminuyendo los impuestos a empresas de alta tecnología o que generan encadenamientos económicos fuertes entre o intra sectorialmente, razón por la cual son de carácter estratégico en las economías nacionales, y no se les debería dejar colapsar, independientemente de su rentabilidad. Otra opción es que los jóvenes de 14 años o más, trabajen a medio tiempo en actividades relacionadas con su aprendizaje, mientras estudian en el colegio, sobre todo si existe adiestramiento en el manejo de software específico para empresas que lo necesitan, en diseño y arte (Azizi \& Lasonen, 
2006; Skelton \& Valentine, 2005), o en locales comerciales y en restaurantes como generalmente sucede en los países más ricos del planeta. Sin embargo se pueden abrir nuevos mercados para gente de la tercera edad en el sector de salud y cuidado de ellos, los mismos que proporcionarán perspectivas de empleo adicionales y crearán negocios para servicios y productos complementarios (Walkenhorst \& Sila, 2015).

Se debe también indicar que en la actualidad se están sustituyendo obreros en fábricas por robots, tal como ha sucedido últimamente con FoxConn y otras compañías (Qureshi \& Syed, 2014), tendencia originada desde comienzos de la década de 1960, cuando General Motors adquirió para sus ensambladoras de carros los primeros robots Unimation (Ayres \& Miller, 1982). Los gobiernos deberían, en vez de preocuparse por el decrecimiento poblacional, más bien prever la existencia de mayores niveles de automatización en el futuro (Pagliarini \& Lund, 2017), no sólo en el sector industrial, pero incluso en el sector agrícola y de servicios, sobre todo si se siguen desarrollando programas de auto-conducción para vehículos como fabrica Tesla Motors y otras empresas (Endsley, 2017; Jones, 2017) o para maquinarias agrícolas y de inteligencia artificial capaces de realizar diagnósticos médicos o de cualquier otro tipo de información en cuestión de pocos minutos como lo realiza la supercomputadora Watson de IBM (McKenna, 2017), o robots capaces de velar por los ancianos (Hebesberger, Koertner, Gisinger \& Pripfl, 2017), lo que implicará consecuentemente menos empleo para los humanos mientras la productividad se incrementará (McClure, 2017).

De hecho, la productividad promedio de la PEA per cápita, excluyendo a los desempleados, no se incrementa porque las personas están más educadas y capacitadas, pues si no hay inversión productiva y generación de empleo, no se consigue nada; el aumento en la productividad se debe más bien a factores relacionados con el tamaño de las Unidades de Producción Agropecuarias (UPAs), el acceso a créditos, a subsidios, a mecanización agrícola, mejores especímenes, a productos fitosanitarios o veterinarios, a abonos y alimentos, y a precios justos en el sector primario. El sector secundario y minero dependen sobre todo de la utilización de maquinaria de alta tecnología para producir a gran escala de manera eficiente, mientras que en el sector terciario la productividad de muchos servicios es más difícil que aumente, ya que ésta depende del conocimiento y de la técnica empleada por personas que trabajan principalmente con herramientas, y sólo al ser éstas reemplazadas con mejores y más modernas, mejorará la productividad por persona (Donoso Correa \& Donoso Correa, 2016). Por otro lado, la pequeña escala y la baja productividad de las tecnologías empleadas en el laboratorio son complicadas muchas veces de cambiar a la gran escala y alta productividad de las plantas industriales (Markham, 2002).

\section{CONCLUSIONES}

Es fundamental comprender los procesos y parámetros básicos que ligan a las variables poblacionales con las económicas (Boserup, 1990; Kelley \& Schmidt, 1995), sobre todo con el PIB y PIB per cápita, debiéndose precisar que los indicadores demográficos son más factibles de ser predichos en el futuro, con mayor exactitud y con menos error que las variables macroeconómicas, por ejemplo, las tasas de crecimiento poblacional siguen el modelo de transición demográfica y no varían tanto de un año a otro, mientras que las tasas de crecimiento del PIB pueden fluctuar drásticamente en el corto plazo.

Todos los países del planeta se encuentran atravesando en la actualidad las últimas tres etapas del modelo de transición demográfica, lo que significa que anteriormente todos ellos debieron haber pasado ya por las dos primeras fases y hasta mediados del siglo 21 todas las naciones culminarán su transición, primero, estabilizando sus poblaciones y luego declinando lentamente. Por esta razón, si los gobiernos de los países que se encuentran en los últimos dos periodos de este modelo, empiezan a pensar, planificar e implementar políticas y normativas, como las expuestas en este trabajo u otros similares, podrán anticiparse, adaptarse y contrarrestar mejor las consecuencias económicas que conlleva el decrecimiento poblacional en los ámbitos de la producción.

Respecto a las consecuencias del declive poblacional en la seguridad social, salud, mercados bursátiles e inmobiliarios, recursos naturales, utilización de energía, pérdida de poder militar y quizá otros efectos más, éstas no han sido mencionadas, consideradas o tratadas en este trabajo investigativo, 
así mismo sería sumamente interesante indagar sobre cómo afecta psicológicamente el tamaño reducido de las nuevas familias a los hijos que crecen sin hermanos y sin tíos, o cómo éstas sociedades de hogares minúsculos van alterando paulatinamente las culturas de las distintas naciones.

\section{REFERENCIAS}

Ashton, T. S. (1997). The industrial revolution 1760-1830. OUP Catalogue. Retrieved from https://ideas.repec.org/b/oxp/obooks/9780192892898.html

Ayres, R., Miller, S. (1982). Industrial robots on the line. The Journal of Epsilon Pi Tau, 8(2), 2-10.

Azizi, N., Lasonen, J. (2006). Education, training and the economy: preparing young people for a changing labour market. Jyväskylä, Finland: Institute for Educational Research, University of Jyväskylä.

Bacci, M. L. (2017). A concise history of world population. John Wiley \& Sons.

Barlow, R. (1994). Population growth and economic growth: Some more correlations. Population and Development Review, 20(1), 153-165.

Becker, G. S. (2002). The age of human capital. Retrieved from https://www.hoover.org/sites/default/files/uploads/documents/0817928928_3.pdf

Becker, G. S., Chiswick, B. R. (1966). Education and the distribution of earnings. The American Economic Review, 56(1/2), 358-369.

Becker, G. S., Murphy, K. M., Tamura, R. (1990). Human capital, fertility, and economic growth. The Journal of Political Economy, 98(5, Part 2), S12-S37.

Berelson, B. R. (1974). Population policy in developed countries. New York: McGraw-Hill.

Birdsall, N., Kelley, A. C., Sinding, S. W. (2001). Population matters: Demographic change, economic growth, and poverty in the developing world. Oxford University Press.

Bloom, D., Canning, D., Sevilla, J. (2003). The demographic dividend: A new perspective on the economic consequences of population change. Rand Corporation: A RAND Program of PolicyRelevant Research Communication, 125 pp.

Bloom, D. E., Canning, D., Fink, G. (2010). Implications of population ageing for economic growth. Oxford Review of Economic Policy, 26(4), 583-612.

Borner, S., Grubel, H. (2016). The European Community after 1992: Perspectives from the Outside. Springer.

Boserup, E. (1990). Economic and demographic relationships in development. Population and Development Review, 16(4), 775-779.

Canning, D. (1998). A database of world stocks of infrastructure, 1950-95. The World Bank Economic Review. Retrieved from http://wber.oxfordjournals.org/content/12/3/529.short

Chapple, J. (2004). The dilemma posed by Japan's population decline. Electronic Journal of Contemporary Japanese Studies. Retrieved from https://www.japanesestudies.org.uk/discussionpapers/Chapple.html

Chesnais, J.-C. (1992). The demographic transition: Stages, patterns, and economic implications: a longitudinal study of sixty-seven countries covering the period 1720-1984. Oxford University Press.

Chiavacci, D., Hommerich, C. (2016). Social inequality in post-growth Japan: Transformation during economic and demographic stagnation. Routledge: Taylor \& Francis Group.

Coale, A. J. (2017). The decline of fertility in Europe. Princeton University Press.

Coleman, D., Rowthorn, R. (2011). Who's afraid of population decline? A critical examination of its consequences. Population and Development Review, 37, 217-248.

Coulmas, F. (2007). Population decline and ageing in Japan - The social consequences. Routledge. 
Cruces, G., Fields, G. S., Viollaz, M., Jaume, D. (2017). Growth, employment, and poverty in Latin America. Oxford University Press.

Daly, M. E. (2006). The slow failure: Population decline and independent Ireland, 1920-1973. University of Wisconsin Pres.

DESA. (2004). World population to 2300. United Nations Department of Economics and Social Affairs, New York, NY [available a T Www. Un. org/esa/population/publications/longrange2/WorldPop2300final. Pdf].

DESA. (2015). World population prospects: The 2015 revision, key findings and advance tables. Retrieved from https://esa.un.org/unpd/wpp/DataQuery/

Donoso Correa, M. E. (2000). Análisis de la evolución demográfica del Ecuador 1950-2000. Retrieved from http://dspace.ucuenca.edu.ec/handle/123456789/15120

Donoso Correa, M. E., Donoso Correa, J. S. (2016). Multi agentes y autómatas celulares como base para el análisis eficaz y realista de la macroeconomía. Retrieved from http://dspace.ucuenca.edu.ec/handle/123456789/26255

Easterlin, R. A. (1967). Effects of population growth on the economic development of developing countries. The Annals of the American Academy of Political and Social Science, 369(1), 98-108.

Easterlin, R. A. (1976). The conflict between aspirations and resources. Population and Development Review, 2(3/4), 417-425.

Endsley, M. R. (2017). Autonomous driving systems: A preliminary naturalistic study of the Tesla model S. Journal of Cognitive Engineering and Decision Making, 1555343417695197.

Faruqee, R., Gulhati, R. (1983). Rapid population growth in Sub-Saharan Africa: issues and policies. Retrieved from https://www.popline.org/node/393075

Gerland, P., Raftery, A. E., Sevčíková, H., Li, N., Gu, D., Spoorenberg, T., Alkema, L., Fosdick, B. K., Chunn, J., Lalic, N., Baly, G., Buettner, T., Heilig, G. K., Wilmoth, J. (2014). World population stabilization unlikely this century. Science, 346(6206), 234-237.

Goldstein, J., Lutz, W., Scherbov, S. (2003). Long-term population decline in Europe: The relative importance of tempo effects and generational length. Population and Development Review, 29(4), 699-707.

Gragnolati, M., Rofman, R., Apella, I., Troiano, S. (2015). As time goes by in Argentina: Economic opportunities and challenges of the demographic transition. World Bank Publications.

Hall, P. A. (1989). The political power of economic ideas: Keynesianism across nations. Retrieved from http://scholar.harvard.edu/hall/publications/political-power-economic-ideas-keynesianismacross-nations

Harrison, M. (2000). The economics of World War II: Six great powers in international comparison. Cambridge University Press.

Hebesberger, D., Koertner, T., Gisinger, C., Pripfl, J. (2017). A long-term autonomous robot at a care hospital: A mixed methods study on social acceptance and experiences of staff and older adults. Advanced Robotics: The International Journal of the Robotics Society of Japan, 1-13.

Helminen, M. (1999). The dividend concept in International Tax Law: Dividend payments between corporate entities. Kluwer Law International.

Hobbs, F., Stoops, N. (2002). Demographic trends in the 20th century. U.S. Census Bureau.

Hogan, M. J. (1987). The Marshall Plan: America, Britain and the reconstruction of Western Europe, 1947-1952. Cambridge University Press.

Horelick, B., Koont, S., Gootlieb, S. F. (1983). Population growth and the logistic curve. Retrieved from https://www.maa.org/press/periodicals/loci/joma/logistic-growth-model-backgroundlogistic-modeling

Huh, H.-S., Lee, H.-H., Lee, Y.-Y. (2007). Demographic transition and its impact on economic growth. In: Korea and the World Economy VI Conference, University of Wollongong, Australia (pp. 2-3). researchgate.net. 
Jones, C. (1995). R \& D-based models of economic growth. The Journal of Political Economy, 103(4), 759-784.

Jones, L. (2017). Driverless cars: when and where? Engineering \& Technology, 12(2), 36-40.

Jorgenson, D. W., Vu, K. M. (2017). The outlook for advanced economies. Journal of Policy Modeling, 39(4), 660-672.

Kaldor, N. (1966). Causes of the slow rate of economic growth of the United Kingdom: an inaugural lecture. Cambridge University Press.

Katus, K. (1991). Fertility transition in Estonia, Latvia and Lithuania. Demographic Trends and Patterns in the Soviet Union before, 89-111.

Katus, K. (1997). Long-term fertility development in Baltoscandia. Yearbook of Population Research in Finland = Vaestontutkimuksen vuosikirja, 34, 18-34.

Keilman, N. (1998). How accurate are the United Nations world population projections? Population and Development Review, 24, 15-41.

Keyfitz, N. (1981). The limits of population forecasting. Population and Development Review, 7(4), 579-593.

Keynes, J. M. (1968). Some economic consequences of a declining population. The Eugenics Review, 60(1), 57-61.

Kuznets, S. (1967). Population and economic growth. Proceedings of the American Philosophical Society, 111(3), 170-193.

Landry, A. (1934). La révolution démographique: études et essais sur les problèmes de la population. France: INED.

Lee, H.-H., Huh, H., Lee, Y.-Y., Lim, J.-Y. (2013). Effects of population aging on economic growth: A panel analysis. Retrieved from https://papers.ssrn.com/sol3/papers.cfm?abstract_id=2364194

Lee, R. (1983). Economic consequences of population size structure and growth. Newsletter: International Union for the Scientific Study of Population, 26(2), 159-182.

Lindh, T. (2005). Productivity is a system property and need not decrease with the age of the workforce. Vienna Yearbook of Population Research / Vienna Institute of Demography, Austrian Academy of Sciences, 3, 7-9.

Loschky, D. J., Wilcox, W. C. (1974). Demographic transition: A forcing model. Demography, 11(2), 215.

Lutz, W., Scherbov, S., Volkov, A. (2002). Demographic trends and patterns in the Soviet Union before 1991. Routledge.

Macpherson, W. (1994). The industrial revolution in Japan. Wiley-Blackwell.

Mantoux, P. (2013). The industrial revolution in the eighteenth century: An outline of the beginnings of the modern factory system in England. Routledge.

Markham, S. K. (2002). Moving technologies from lab to market. Research-Technology Management, 45(6), 31-42.

Mason, A. (2005). Demographic transition and demographic dividends in developed and developing countries. In: United Nations expert group meeting on social and economic implications of changing population age structures (Vol. 31). Citeseer. Retrieved from http://citeseerx.ist.psu.edu/viewdoc/download?doi=10.1.1.365.2298\&rep=rep1\&type=pdf\#page= 95

Matsutani, A. (2006). Shrinking-population economics: Lessons from Japan. LTCB International Library Trust.

Mauldin, W. P. (1976). Fertility trends: 1950-75. Studies in Family Planning, 7(9), 242-248.

McClure, P. K. (2017). “You're fired,” says the robot: The rise of automation in the workplace, technophobes, and fears of unemployment. Social Science Computer Review, 0894439317698637.

McCormick, A. L. (2014). The industrial revolution in United States history. Enslow Publishers. 
McKenna, J. (2017). Big data: big promise. European Heart Journal, 38(7), 470-471.

Mokyr, J., Grada, C. O. (1984). New developments in Irish population history, 1700-1850. The Economic History Review, 37(4), 473.

Morgan, P. (2003). Is low fertility a twenty-first-century demographic crisis? Demography, 40(4), 589-603.

Musson, A. E., Robinson, E. (1969). Science and technology in the industrial revolution. Manchester University Press.

National Research Council. (1986). Population growth and economic development: Policy questions. National Academies Press.

Newbigin, M. I. (1918). Some aspects of the industrial revolution in Western Europe. Scottish Geographical Magazine, 34(7), 251-263.

OECD data. (2017). Retrieved from https://data.oecd.org/

Ogawa, N., Mason, A., Chawla, A., Matsukura, R., Tung, A.-C. (2009). Declining fertility and the rising cost of children. Asian Population Studies, 5(3), 289-307.

Pagliarini, L., Lund, H. H. (2017). The future of robotics technology. Journal of Robotics Networking and Artificial Life, 3(4), 270-273.

Partida-Bush, V. (2004). How demographic transition forms demographic bonus and aging process in Mexico. Séminaire Les Transitions Des Structures Par âge: Bonus Démographique, Mais Défis Nouveaux Pour La Population et Le Développement Durable, CICRED, Paris, 23-26.

Pe'er, A., Vertinsky, I., Keil, T. (2016). Growth and survival: The moderating effects of local agglomeration and local market structure. Strategic Management Journal, 37(3), 541-564.

Poterba, J. (2016). Economic implications of demographic change. Business Economics, 51(1), 3-7.

Poulalion, G. (1984). The science of population. Retrieved from http://www.popline.org/node/419031

Prais, S. J., Leibenstein, H., Notestein, F. (1955). A theory of economic-demographic development. Population Studies, 9(1), 107.

Prettner, K. (2013). Population aging and endogenous economic growth. Journal of Population Economics, 26(2), 811-834.

Qureshi, M. O., Syed, R. S. (2014). The impact of robotics on employment and motivation of employees in the service sector, with special reference to health care. Safety and Health at Work, 5(4), 198-202.

Roser, M. (2016a). Demographic transition model. Retrieved from https://ourworldindata.org/futureworld-population-growth/

Roser, M. (2016b). Fertility rates in the world 1955-2015. Retrieved from https://ourworldindata.org/future-world-population-growth/

Roser, M. (2016c). Future world population growth. Retrieved from https://ourworldindata.org/future-world-population-growth/

Sauvy, A. (1969). General theory of population. New York, Basic Books.

Simon, J. L. (1989). On aggregate empirical studies relating population variables to economic development. Population and Development Review, 15(2), 323-332.

Simon, J. L., Bartlett, A. A. (1985). The ultimate resource. American Journal of Physics, 53(3), 282-286.

Simon, J. L., Kahn, H. (1984). The resourceful earth: a response to Global 2000. Retrieved from http://www.popline.org/node/407550

Skelton, T., Valentine, G. (2005). Cool places: Geographies of youth cultures. Routledge.

Skirbekk, V. (2005). Population ageing negatively affects productivity (Vol. 2005). Vienna Yearbook of Population Research 2005, Verlag der Österreichischen Akademie der Wissenschaften.

Spéder, Z., Kamarás, F. (2008). Hungary: Secular fertility decline with distinct period fluctuations. Demographic Research, 19(18), 599-664. 
Teitelbaum, M. S. (1975). Relevance of demographic transition theory for developing countries. Science, 188(4187), 420-425.

Thomlinson, R. (1965). Population dynamics causes and consequences of world demographic change. Retrieved from http://www.popline.org/node/520510

Thompson, W. S. (1929). Population. The American Journal of Sociology, 34(6), 959-975.

Turra, C. M., Queiroz, B. L. (2005). Before it’s too late: demographic transition, labor supply and social security problems in Brazil. In: United Nations Expert Group Meeting on Social and Economic Implications of Changing Population Age Structures. un.org. Retrieved from http://docs.google.com/viewer?url=http://www.un.org/en/development/desa/population/publicati ons/pdf/ageing/egm-mex-full-report.pdf\#page $=117$

UNECE. (2002). Economic survey of Europe. United Nations Publications.

Van Bavel, J., Reher, D. S. (2013). The baby boom and its causes: What we know and what we need to know. Population and Development Review, 39(2), 257-288.

Walkenhorst, P., Sila, U. (2015). The economic consequences of an ageing population in Slovenia. Retrieved from http://www.oecd-ilibrary.org/economics/the-economic-consequences-of-anageing-population-in-slovenia_5jrxml4b6828-en

WB. (2017). Fertility rate, total (births per woman): Data. Retrieved from http://data.worldbank.org/indicator/SP.DYN.TFRT.IN

Yamamura, K. (1967). Economic policy in postwar Japan: Growth versus economic democracy. University of California Press.

Yenilmez, M. I. (2015). Economic and social consequences of population aging: The dilemmas and opportunities in the twenty-first century. Applied Research in Quality of Life, 10(4), 735-752. 